\title{
CAUCHY PROBLEM FOR HYPERBOLIC OPERATORS WITH TRIPLE CHARACTERISTICS OF VARIABLE MULTIPLICITY
}

\author{
ENRICO BERNARDI, ANTONIO BOVE AND VESSELIN PETKOV
}

\begin{abstract}
We study a class of third order hyperbolic operators $P$ in $G=\Omega \cap\{0 \leq t \leq T\}, \Omega \subset$ $\mathbb{R}^{n+1}$ with triple characteristics on $t=0$. We consider the case when the fundamental matrix of the principal symbol for $t=0$ has a couple of non vanishing real eigenvalues and $P$ is strictly hyperbolic for $t>0$. We prove that $P$ is strongly hyperbolic, that is the Cauchy problem for $P+Q$ is well posed in $G$ for any lower order terms $Q$.
\end{abstract}

\section{INTRODUCTION}

Consider a differential operator

$$
P\left(t, x, D_{t}, D_{x}\right)=\sum_{\alpha+|\beta| \leq m} c_{\alpha, \beta}(t, x) D_{t}^{\alpha} D_{x}^{\beta}, D_{t}=-\mathbf{i} \partial_{t}, D_{x_{j}}=-\mathbf{i} \partial_{x_{j}}
$$

of order $m$ with $C^{\infty}$ coefficients $c_{\alpha, \beta}(t, x), t \in \mathbb{R}, x \in \mathbb{R}^{n}$. Denote by

$$
p_{m}(t, x, \tau, \xi)=\sum_{\alpha+|\beta|=m} c_{\alpha, \beta}(t, x) \tau^{\alpha} \xi^{\beta}
$$

the principal symbol of $P$. Let $\Omega \subset \mathbb{R}^{n+1}$ be an open set and let

$$
\Omega_{\eta}^{-}=\Omega \cap\{t \leq \eta\}, \Omega_{\eta}^{+}=\Omega \cap\{t \geq \eta\}, G=\Omega \cap\{0 \leq t \leq T\} .
$$

We say that $P$ is hyperbolic with respect to $N_{0}=(1,0, \ldots, 0)$ at $\left(t_{0}, x_{0}\right)$ if

$$
\text { (i) } p_{m}\left(t_{0}, x_{0}, N_{0}\right) \neq 0 \text {, }
$$

(ii) the equation

$$
p_{m}\left(t_{0}, x_{0}, \tau, \xi\right)=0
$$

with respect to $\tau$ has only real roots $\tau=\lambda_{j}\left(t_{0}, x_{0}, \xi\right)$ for all $\xi \in \mathbb{R}^{n}$. Set $P_{m}\left(t, x, D_{t}, D_{x}\right)=$ $p_{m}\left(t, x, D_{t}, D_{x}\right)$.

Definition 1. We say that the Cauchy problem

$$
P u=f \text { in } \Omega \cap\{t<T\} \text {, supp } u \subset \bar{G}
$$

is well posed in $G$ if

(i) (existence) for every $f \in C_{0}^{\infty}(\Omega)$, $\operatorname{supp} f \subset \overline{\Omega_{T}^{-}}$there exists a solution $u \in \mathcal{E}^{\prime}(\Omega)$ satisfying (1.2).

(ii) (uniqueness) if $u \in \mathcal{E}^{\prime}(\Omega)$ satisfies (1.2), then for every $s, 0<s \leq T$, if $P u=0$ in $\Omega_{s}^{-}$, then $u=0$ in $\Omega_{s}^{-}$.

A necessary condition for the well posedeness of the Cauchy problem (WPC) is the hyperbolicity of the operator $P$ at every point $(t, x) \in G$. 
Definition 2. We say that the operator $P$ with principal symbol $p_{m}$ is strongly hyperbolic in $G$ if for every point $z_{0}=\left(t_{0}, x_{0}\right) \in G$ there exists a neighborhood $U$ of $z_{0}$ and $T_{0} \geq 0\left(T_{0}<t_{0}\right.$ if $t_{0}>0$ and $T_{0}=0$ if $t_{0}=0$ ) such that the Cauchy problem (1.2) for the operator $L=$ $P_{m}\left(t, x, D_{t}, D_{x}\right)+Q_{m-1}\left(t, x, D_{t}, D_{x}\right)$ is well posed in $U_{s}^{+}$for every $T_{0} \leq s<T(U)$ and for any operator $Q_{m-1}\left(t, x, D_{t}, D_{x}\right)$ of order less or equal to $m-1$.

A classical result says that if $P$ is strictly hyperbolic, that is the equation (1.1) has simple roots $\lambda_{j}(t, x, \xi)$ for all $(t . x, \xi) \in G \times \mathbb{R}^{N} \backslash\{0\}$, then $P$ is strongly hyperbolic. If the equation (1.1) has real roots with constant multiplicity for $(t, x, \xi) \in G \times \mathbb{R}^{n} \backslash\{0\}$, the operator $P$ is strongly hyperbolic if and only if it is strictly hyperbolic. Thus if we have some roots with constant multiplicity $m_{j} \geq 2$ for the (WPC) we must impose some conditions on lower terms $Q_{m-1}$ called Levi conditions. The analysis of the Cauchy problem for such operators is complete and we know the necessary [4] and sufficient [3] conditions for (WPC).

Passing to the case of variable multiplicity of the roots of (1.1), notice that the roots $\lambda_{j}(t, x, \xi)$ in general are not smooth but only continuous. The case of operators with constant coefficients is also completely examined and $P$ is strongly hyperbolic if and only if $P$ is strictly hyperbolic. The necessary and sufficient condition of Gårding for (WPC) says that there exists a constant $c>0$ such that for the full symbol $p$ of $P$ we have

$$
p(\tau, \xi) \neq 0, \text { for }|\operatorname{Im} \tau|>c, \forall \xi \in \mathbb{R}^{n} .
$$

To understand the situation of variable multiplicity and variable coefficients, consider the example

$$
P=D_{t}^{2}-a(z) D_{x}^{2}+b_{0}(z) D_{t}+b_{1}(z) D_{x}+c(z), z=(t, x) \in \mathbb{R}^{2}
$$

with $a(z) \geq 0$. If $a\left(z_{0}\right)=d a\left(z_{0}\right)=a_{t t}\left(z_{0}\right)=0, b_{1}\left(z_{0}\right) \neq 0$, in a point $z_{0} \in G$, the Cauchy problem for $P$ is not well posed. On the other hand, if for a point $z_{0}=\left(t_{0}, x_{0}\right) \in G$, we have $a\left(z_{0}\right)=d a\left(z_{0}\right)=0, a_{t t}\left(z_{0}\right) \neq 0$, then there exists a neighborhood $U$ of $z_{0}$ such that the Cauchy problem in $U_{t_{0}}^{+}$is well posed for arbitrary smooth lower order terms [14] and $u \in H^{k+2}(U)$ if $f \in H^{k+N}\left(\mathbb{R}^{2}\right), k \in N$, where

$$
N=3+2\left[\frac{3}{2}+\left|b_{1}\left(z_{0}\right)\left(a_{t t}\left(z_{0}\right)\right)^{-1 / 2}\right|\right]
$$

$[z]$ being the integer part of $z$.

Below we change the notations and we denote $t=x_{0}, x=\left(x_{0}, x_{1}, \ldots, x_{n}\right) \in \mathbb{R}^{n+1}$. The dual variables will be denoted by $\xi=\left(\xi_{0}, \xi_{1}, \ldots, \xi_{n}\right)=\left(\xi_{0}, \xi^{\prime}\right)$. Let $\Sigma(p)=\left\{z \in T^{*} \Omega \backslash\{0\}: p(z)=\right.$ $0\}, \Sigma_{1}(p)=\left\{z \in T^{*}(\Omega): z \in \Sigma(p), d p(z)=0\right\}$. If we have a critical point $(\hat{x}, \hat{\xi}) \in \Sigma_{1}(p)$, then the Hamiltonian system

$$
\frac{d x}{d s}=\partial_{\xi} p, \frac{d \xi}{d s}=-\partial_{x} p
$$

has a stationary point and we consider the differential of the right hand part. Thus we obtain the fundamental matrix

$$
F_{p}(\hat{x}, \hat{\xi})=\left(\begin{array}{cc}
p_{\xi, x}(\hat{x}, \hat{\xi}) & p_{\xi, \xi}(\hat{x}, \hat{\xi}) \\
-p_{x, x}(\hat{x}, \hat{\xi}) & -p_{x, \xi}(\hat{x}, \hat{\xi})
\end{array}\right) .
$$

We note below two properties of $F_{p}$ :

1. For every point $z \in \Sigma_{1}(p)$ the Hessian $Q_{p}(X, Y), X, Y \in T_{z}\left(T^{*}(\Omega)\right)$ at $z$ of $\frac{p}{2}$ is well defined. 
Then $Q_{p}(X, Y)=\sigma\left(X, F_{p}(z) Y\right), \sigma$ being the symplectic form on $T^{*}(\Omega)$. Thus after canonical transformation the fundamental matrix is transformed into a similar one and its eigenvalues are invariant under canonical transformations. Hörmander [5] called $F_{p}(z)$ Hamiltonian map of $Q_{p}$.

2. If $P$ is hyperbolic in $G$ and $(\hat{x}, \hat{\xi})$ is a critical point of $p_{m}(x, \xi)$, then $F_{p_{m}}(\hat{x}, \hat{\xi})$ has at most two non vanishing real simple eigenvalues $\mu$ and $-\mu$ and all other eigenvalues $\lambda$ are purely imaginary, that is $\operatorname{Re} \lambda=0$.

The existence of non vanishing real eigenvalues of $F_{p_{m}}(\hat{x}, \hat{\xi})$ is a necessary condition for strong hyperbolicity. More precisely, let $p_{m-1}(x, \xi)=\sum_{|\alpha|=m-1} c_{\alpha}(x) \xi^{\alpha}$ and let

$$
p_{m-1}^{\prime}(x, \xi)=p_{m-1}(x, \xi)+\frac{\mathbf{i}}{2} \sum_{j=0}^{n} \frac{\partial^{2} p_{m}}{\partial x_{j} \partial \xi_{j}}(x, \xi)
$$

be the subprincipal symbol of $P$ which is invariantly defined for $(x, \xi) \in \Sigma_{1}\left(p_{m}\right)$. Then we have the following

Theorem 1 ([7]). If $P$ is strongly hyperbolic in $G$, then at every point $(\hat{x}, \hat{\xi}) \in \Sigma_{1}\left(p_{m}\right)$ the fundamental matrix $F_{p_{m}}(\hat{x}, \hat{\xi})$ has two non-zero real eigenvalues. Moreover, for $\left(x, \xi^{\prime}\right) \in \stackrel{\circ}{G} \times\left(\mathbb{R}^{n} \backslash\{0\}\right)$ the multiplicities of the roots of (1) are not greater than two, and for $\left(x, \xi^{\prime}\right) \in\left\{x_{0}=0\right\} \times \mathbb{R}^{n} \backslash\{0\}$ or for $\left(x, \xi^{\prime}\right) \in\left\{x_{0}=T\right\} \times \mathbb{R}^{n} \backslash\{0\}$ these multiplicities are not greater than three. If $F_{p_{m}}(\hat{x}, \hat{\xi})$ has only purely imaginary eigenvalues, the condition $\operatorname{Im} p_{m-1}^{\prime}(\hat{x}, \hat{\xi})=0$ is necessary for $(W P C)$.

If $F_{p_{m}}(\hat{x}, \hat{\xi})$ has only purely imaginary eigenvalues, for (WCP) we have a second necessary condition

$$
\left|\operatorname{Re} p_{m-1}^{\prime}(\hat{x}, \hat{\xi})\right| \leq \frac{1}{4} \sum_{j=0}^{2 n+2}\left|\mu_{j}\right|,
$$

$\mu_{j}$ being the eigenvalues of $F_{p_{m}}(\hat{x}, \hat{\xi})$ repeated following their multiplicities. This condition has been proved in [7] in some special cases concerning the structure of $F_{p_{m}}(\hat{x}, \hat{\xi})$ and without any restriction by Hörmander [5].

Definition 3. A hyperbolic operator with principal symbol $p(x, \xi)$ will be called effectively hyperbolic if at every point $(\hat{x}, \hat{\xi}) \in \Sigma_{1}(p)$, the fundamental matrix $F_{p}(\hat{x}, \hat{\xi})$ has two non-zero real eigenvalues.

$\mathrm{V}$. Ivrii introduced the following

Conjecture A hyperbolic operator is strongly hyperbolic if and only if it is effectively hyperbolic.

For operators with at most double characteristics some results for special class of operators have been obtained by Hörmander [5], Ivrii [8] and Melrose [11]. The sufficient part of the above conjecture is difficult since the double roots of the equation (1) in general are not smooth and we have not a factorization with smooth factors. Moreover, the loss of regularity could depend on the point and a microlocalization leads to considerable difficulties when we must treat the commutators. The above conjecture for operators with double characteristics has been completely solved by N. Iwasaki [9], [10] and T. Nishitani [12], [13]. The proofs are rather long and very technical.

An effectively hyperbolic operator could be strongly hyperbolic if it has triple characteristics on the boundary on $G$ but to our best knowledge there are no examples of such operators in the 
literature. Our purpose is to study a class of operators $P$ with triple characteristics on $t=0$ and to prove that $P$ is strongly hyperbolic. Thus the above conjecture is true for some special operators with triple characteristics. The analysis of the general case remains open.

\section{HyPERBoliC OPERATORS WITH TRIPLE CHARACTERISTICS}

In this section we use again the notations of Section 1. According to Theorem 1, an effectively hyperbolic operator $P$ in $G$ may have triple characteristics in $G$ only for $t=0$ or $t=T$. Assume that $P$ has triple characteristics for $t=0$ and suppose that the triple roots of (1.1) for $t=0$ are $\tau=0$ (in general the triple characteristics for $t=0$ are $\tau=\lambda(0, x, \xi)$ ). Let $P$ be of order 3 and let

$$
p_{3}=\tau^{3}+q_{1}(t, x, \xi) \tau^{2}+q_{2}(t, x, \xi) \tau+q_{3}(t, x, \xi)
$$

be the principal symbol of $P$ with $q_{j}, j=1,2,3$, real-valued polynomials of order $j$ with respect to $\xi$ with smooth coefficients.

Lemma 1 ([7]). Let $p_{3}(t, x, \tau, \xi)$ be hyperbolic in $G$ and let $\tau=0$ be a triple root of $p_{3}(0, x, \tau, \xi)=$ $0,(0, x) \in G$. Then

$$
q_{3}(0, x, \xi)=\partial_{t} q_{3}(0, x, \xi)=q_{2}(0, x, \xi)=q_{1}(0, x, \xi)=0,(0, x) \in G, \xi \in \mathbb{R}^{n} .
$$

Moreover, $p_{3}$ is effectively hyperbolic for $t=\tau=0$, if and only if

$$
\frac{\partial^{2} p_{3}}{\partial \tau \partial t}(0, x, 0, \xi)<0, \xi \in \mathbb{R}^{n} \backslash\{0\} .
$$

Thus we must study an operator $P$ with principal part

$$
P_{3}=D_{t}^{3}+t a_{1}\left(t, x, D_{x}\right) D_{t}^{2}-t a_{2}\left(t, x, D_{x}\right) D_{t}+t^{2} a_{3}\left(t, x, D_{x}\right)
$$

with $a_{j}(t, x, \xi)$ real-valued polynomials of order $j$ in $\xi$ and $a_{2}(t, x, \xi) \geq c|\xi|^{2}, c>0$ for $\xi \neq 0$. We write $P=P_{3}+Q$ with lower order terms $Q=B_{2}\left(t, x, D_{x}\right)+B_{1}\left(t, x, D_{x}\right) D_{t}+C\left(t, x, D_{t}, D_{x}\right)$. Here $B_{2}$ and $B_{1}$ are differential operator of order 2 and 1 , respectively, while $C$ is an operator of order 1. Notice that for $|\xi|=1$ the discriminant $\Delta$ of the equation $p_{3}(t, x, \tau, \xi)=0$ with respect to $\tau$ has the form

$$
\begin{gathered}
\Delta(t, x, \xi)=\left(\frac{-3 t a_{2}-t^{2} a_{1}^{2}}{9}\right)^{3}+\left(\frac{-9 t^{2} a_{1} a_{2}-27 t^{2} a_{3}-2 t^{3} a_{1}^{3}}{54}\right)^{2} \\
=q^{3}+r^{2}=-\frac{1}{27} t^{3} a_{2}^{3}+\mathcal{O}\left(t^{4}\right) a_{6}
\end{gathered}
$$

and $\Delta \leq 0$ for small $t \geq 0$. Thus the operator $P$ is strictly hyperbolic for small $t>0$ and it suffices to examine the Cauchy problem for $0 \leq t \leq t_{0}, t_{0} \ll 1$. Since the coefficients of the cubic equation $p_{3}(t, x, \tau, \xi)=0$ are real, for $t \geq 0$ its real roots $\lambda_{k}(t, x, \xi), k=1,2,3$, have the following trigonometric form (see for instance, [15])

$$
\left\{\begin{array}{l}
\lambda_{1}=2 \rho^{1 / 3} \cos (\theta / 3)-\frac{t a_{1}}{3} \\
\lambda_{2}=2 \rho^{1 / 3} \cos \left(\theta / 3+\frac{2 \pi}{3}\right)-\frac{t a_{1}}{3} \\
\lambda_{3}=2 \rho^{1 / 3} \cos \left(\theta / 3+\frac{4 \pi}{3}\right)-\frac{t a_{1}}{3}
\end{array}\right.
$$

where

Next consider the symbols

$$
\rho=(-q)^{3 / 2}, \theta=\arccos (r / \rho)
$$

$$
\delta_{k}=\left.\frac{\partial p_{3}}{\partial \tau}\right|_{\tau=\lambda_{k}}=\left.\left(3 \tau^{2}+2 t a_{1} \tau-t a_{2}\right)\right|_{\tau=\lambda_{k}}, k=1,2,3 .
$$


Since these symbols are homogeneous of order 2 in $\xi$, to find lower bounds for $\left|\delta_{k}\right|$, it is sufficient to examine their behavior for $|\xi|=1$. We have

$$
\delta_{1}=12 \rho^{2 / 3} \cos ^{2}(\theta / 3)-t a_{2}+\mathcal{O}\left(t^{3 / 2}\right) a_{2}=\left(4 \cos ^{2}(\theta / 3)-1\right) t a_{2}+\mathcal{O}\left(t^{3 / 2}\right) a_{2} .
$$

Since $\frac{r}{\rho}=\mathcal{O}\left(t^{1 / 2}\right)$, we have $\cos (\theta / 3)=\frac{\sqrt{3}}{2}+o(t)$ and this implies for small $t$ and $|\xi|=1$ the estimate $\left|\delta_{1}\right| \geq c_{1} t a_{2}$ with $c_{1}>0$. On the other hand,

$$
\delta_{2,3}=3 \lambda_{2,3}^{2}-t a_{2}+\mathcal{O}\left(t^{3 / 2}\right) a_{2}=\left(4 \sin ^{2}(\pi / 6 \pm \theta / 3)-1\right) t a_{2}+\mathcal{O}\left(t^{3 / 2}\right) a_{2}
$$

and we obtain the following

Lemma 2. There exist constants $\gamma>0$ and $\gamma_{1}>0$ such that for $0 \leq t \leq \gamma_{1}$ we have

$$
\left|\delta_{k}\right| \geq \gamma t a_{2}(t, x, \xi) \geq \gamma c t|\xi|^{2}, k=1,2,3 \text {. }
$$

Finally, notice that $\lambda_{1} \lambda_{2} \lambda_{3}=-t^{2} a_{3}(t, x, \xi)$.

\section{ENERGY ESTIMATES FOR A MODEL OPERATOR}

Consider the operator

$$
P\left(t, D_{t}, D_{x}\right)=D_{t}^{3}+t a_{1}\left(t, D_{x}\right) D_{t}^{2}-t a_{2}\left(D_{x}\right) D_{t}+t^{2} a_{3}\left(t, D_{x}\right)+b\left(t, D_{x}\right), t \geq 0
$$

where $a_{2}\left(D_{x}\right)=\sum_{i, j=1}^{n} a_{i, j} D_{i} D_{j}$ and $b\left(t, D_{x}\right)=\sum_{i, j=1}^{n} b_{i, j}(t) D_{i} D_{j}$ is a second order differential operator. For simplicity we assume that $a_{2}$ is independent on $t$. The analysis of operators with $a_{2}\left(t, D_{x}\right)$ goes without any change. We assume that

$$
a(\xi)=a_{2}(\xi)=\sum_{i, j=1}^{n} a_{i, j} \xi_{i} \xi_{j} \geq \delta_{0}|\xi|^{2}, \delta_{0}>0 .
$$

Moreover, the symbols $a_{1}(t, \xi), a_{3}(t, \xi)$ are real-valued and homogeneous of order 1 and 3 in $\xi$, respectively. We want to establish an a priori estimate for $P$ for $t \geq 0$. Set

$$
f(t, \xi)=t+\frac{1}{(1+a(\xi))^{1 / 3}} .
$$

Let $v(t, x) \in C_{0}^{\infty}\left(\mathbb{R}_{t} \times \mathbb{R}^{n}\right)$. Multiplying $P$ by $-\mathbf{i}$ and taking the Fourier transform with respect to the variable $x$, we obtain

$$
\hat{P} u=\widehat{-\mathbf{i} P v}=\partial_{t}^{3} u+\mathbf{i} t a_{1}(t, \xi) u^{\prime \prime}+t \partial_{t} a(\xi) u-\mathbf{i} t^{2} a_{3}(t, \xi) u+b_{1}(t, \xi) u
$$

with $b_{1}\left(t, D_{x}\right)=-\mathbf{i} b\left(t, D_{x}\right)$ and $u=\hat{v}$. Let $u^{\prime \prime}=\widehat{v_{t t}}, u^{\prime}=\hat{v}_{t}$. We have

$$
2 \operatorname{Re} \hat{P} u \bar{u}^{\prime \prime}=\partial_{t}\left|u^{\prime \prime}\right|^{2}+t a(\xi) \partial_{t}\left|u^{\prime}\right|^{2}+2 t^{2} a_{3}(t, \xi) \operatorname{Im}\left(u \bar{u}^{\prime \prime}\right)+2 \operatorname{Re}\left(b_{1}(t, \xi) u \bar{u}^{\prime \prime}\right) .
$$

Denote by $N$ a large positive integer and by $\lambda$ a large positive parameter. Multiply the above identity involving $\hat{P} u$ by $e^{-\lambda t} f^{-2 N}$. We obtain

$$
\begin{aligned}
e^{-\lambda t} f^{-2 N} 2 \operatorname{Re}\left(\hat{P} u \bar{u}^{\prime \prime}\right) & =e^{-\lambda t} f^{-2 N} \partial_{t}\left|u^{\prime \prime}\right|^{2}+e^{-\lambda t} f^{-2 N} t a(\xi) \partial_{t}\left|u^{\prime}\right|^{2} \\
& +e^{-\lambda t} f^{-2 N} 2\left(t^{2} a_{3}(t, \xi) \operatorname{Im}\left(u \bar{u}^{\prime \prime}\right)+\operatorname{Re} b_{1}(t, \xi) u \bar{u}^{\prime \prime}\right) \\
=e^{-\lambda t} f^{-2 N} \partial_{t} \tilde{E}(u) & -e^{-\lambda t} f^{-2 N} a(\xi)\left|u^{\prime}\right|^{2}+e^{-\lambda t} f^{-2 N} 2\left(t^{2} a_{3}(t, \xi) \operatorname{Im}\left(u \bar{u}^{\prime \prime}\right)+\operatorname{Re} b_{1}(t, \xi) u \bar{u}^{\prime \prime}\right),
\end{aligned}
$$


where

$$
\tilde{E}(u)=\left|u^{\prime \prime}\right|^{2}+t a(\xi)\left|u^{\prime}\right|^{2} .
$$

The above identity can be rewritten as

$$
\begin{aligned}
& e^{-\lambda t} f^{-2 N} 2 \operatorname{Re}\left(\hat{P} u \bar{u}^{\prime \prime}\right)=\partial_{t}\left(e^{-\lambda t} f^{-2 N} \tilde{E}(u)\right)+\lambda e^{-\lambda t} f^{-2 N} \tilde{E}(u) \\
& +2 N e^{-\lambda t} f^{-2 N-1} \tilde{E}(u)-e^{-\lambda t} f^{-2 N} a(\xi)\left|u^{\prime}\right|^{2}+2 e^{-\lambda t} f^{-2 N}\left(t^{2} a_{3}(t, \xi) \operatorname{Im}\left(u \bar{u}^{\prime \prime}\right)+\operatorname{Re} b_{1}(t, \xi) u \bar{u}^{\prime \prime}\right) .
\end{aligned}
$$

Since

$$
e^{-\lambda t} f^{2 N} 2 \operatorname{Re}\left(\hat{P} u \bar{u}^{\prime \prime}\right) \leq e^{-\lambda t} f^{-2 N+1}|\hat{P} u|^{2}+e^{-\lambda t} f^{-2 N-1}\left|u^{\prime \prime}\right|^{2},
$$

we have the inequality

$$
\begin{aligned}
e^{-\lambda t} f^{-2 N+1}|\hat{P} u|^{2} \geq \partial_{t} & \left(e^{-\lambda t} f^{-2 N} \tilde{E}(u)\right)+\lambda e^{-\lambda t} f^{-2 N} \tilde{E}(u) \\
+ & (2 N-1) e^{-\lambda t} f^{-2 N-1}\left|u^{\prime \prime}\right|^{2}+2 N e^{-\lambda t} f^{-2 N-1} t a(\xi)\left|u^{\prime}\right|^{2} \\
& -e^{-\lambda t} f^{-2 N} a(\xi)\left|u^{\prime}\right|^{2}+2 e^{-\lambda t} f^{-2 N}\left(t^{2} a_{3}(t, \xi) \operatorname{Im}\left(u \bar{u}^{\prime \prime}\right)+\operatorname{Re} b_{1}(t, \xi) u \bar{u}^{\prime \prime}\right) .
\end{aligned}
$$

Let us now consider the following identity, where $k$ is a positive integer and $g$ denotes a smooth function in the same class as $u$ :

$$
e^{-\lambda t} f^{-2 k} 2 \operatorname{Re} g^{\prime} \bar{g}=\partial_{t}\left(e^{-\lambda t} f^{-2 k}|g|^{2}\right)+\lambda e^{-\lambda t} f^{-2 k}|g|^{2}+2 k e^{-\lambda t} f^{-2 k-1}|g|^{2} .
$$

This implies

$$
e^{-\lambda t} f^{-2 k+1}\left|g^{\prime}\right|^{2} \geq \partial_{t}\left(e^{-\lambda t} f^{-2 k}|g|^{2}\right)+\lambda e^{-\lambda t} f^{-2 k}|g|^{2}+(2 k-1) e^{-\lambda t} f^{-2 k-1}|g|^{2} .
$$

Now, taking $g=u^{\prime}$ we have

$$
e^{-\lambda t} f^{-2 k+1}\left|u^{\prime \prime}\right|^{2} \geq \partial_{t}\left(e^{-\lambda t} f^{-2 k}\left|u^{\prime}\right|^{2}\right)+\lambda e^{-\lambda t} f^{-2 k}\left|u^{\prime}\right|^{2}+(2 k-1) e^{-\lambda t} f^{-2 k-1}\left|u^{\prime}\right|^{2},
$$

while, taking $g=u$, we get

$$
e^{-\lambda t} f^{-2 k+1}\left|u^{\prime}\right|^{2} \geq \partial_{t}\left(e^{-\lambda t} f^{-2 k}|u|^{2}\right)+\lambda e^{-\lambda t} f^{-2 k}|u|^{2}+(2 k-1) e^{-\lambda t} f^{-2 k-1}|u|^{2} .
$$

From (3.2) and (3.3) above we obtain

$$
\begin{aligned}
e^{-\lambda t} f^{-2 k+1}\left|u^{\prime \prime}\right|^{2} \geq \partial_{t}\left(e^{-\lambda t} f^{-2 k}\left|u^{\prime}\right|^{2}\right)+\lambda e^{-\lambda t} f^{-2 k}\left|u^{\prime}\right|^{2} & \\
& +(2 k-2) e^{-\lambda t} f^{-2 k-1}\left|u^{\prime}\right|^{2} \\
& +\partial_{t}\left(e^{-\lambda t} f^{-2 k-2}|u|^{2}\right)+\lambda e^{-\lambda t} f^{-2 k-2}|u|^{2}+(2 k+1) e^{-\lambda t} f^{-2 k-3}|u|^{2}
\end{aligned}
$$


Plugging this into the estimate for $|\hat{P} u|^{2}$ and choosing $k=N+1$, we obtain

$$
\begin{gathered}
e^{-\lambda t} f^{-2 N+1}|\hat{P} u|^{2} \geq \partial_{t}\left(e^{-\lambda t} f^{-2 N} \tilde{E}(u)\right)+\lambda e^{-\lambda t} f^{-2 N} \tilde{E}(u) \\
+\mathcal{O}(N)\left\{e^{-\lambda t} f^{-2 N-1}\left|u^{\prime \prime}\right|^{2}+\partial_{t}\left(e^{-\lambda t} f^{-2 N-2}\left|u^{\prime}\right|^{2}\right)+\lambda e^{-\lambda t} f^{-2 N-2}\left|u^{\prime}\right|^{2}\right\} \\
+\mathcal{O}\left(N^{2}\right) e^{-\lambda t} f^{-2 N-3}\left|u^{\prime}\right|^{2} \\
+\mathcal{O}(N)\left\{\partial_{t}\left(e^{-\lambda t} f^{-2 N-4}|u|^{2}\right)+\lambda e^{-\lambda t} f^{-2 N-4}|u|^{2}\right\} \\
+\mathcal{O}\left(N^{2}\right) e^{-\lambda t} f^{-2 N-5}|u|^{2} \\
+2 N e^{-\lambda t} f^{-2 N-1} t a(\xi)\left|u^{\prime}\right|^{2}-e^{-\lambda t} f^{-2 N} a(\xi)\left|u^{\prime}\right|^{2} \\
+2 e^{-\lambda t} f^{-2 N}\left(t^{2} a_{3}(t, \xi) \operatorname{Im}\left(u \bar{u}^{\prime \prime}\right)+\operatorname{Re} b_{1}(t, \xi) u \bar{u}^{\prime \prime}\right) .
\end{gathered}
$$

Here $\mathcal{O}(N)$ means a function of $N$ which satisfies an estimate of the type: $\mathcal{O}(N) \geq c N$, with a fixed positive constant $c$.

From inequality (3.4) above we also deduce that

$$
\begin{aligned}
e^{-\lambda t} f^{-2 N-1} t a(\xi)\left|u^{\prime}\right|^{2} \geq \partial_{t}\left(e^{-\lambda t} f^{-2 N-2}\right. & \left.t a(\xi)|u|^{2}\right)+\lambda e^{-\lambda t} f^{-2 N-2} t a(\xi)|u|^{2} \\
& -e^{-\lambda t} f^{-2 N-2} a(\xi)|u|^{2}+(2 N+1) e^{-\lambda t} f^{-2 N-3} t a(\xi)|u|^{2} .
\end{aligned}
$$

Replacing the part of the corresponding term in (3.12) with the above inequality, we finally obtain

$$
\begin{gathered}
e^{-\lambda t} f^{-2 N+1}|\hat{P} u|^{2} \geq \partial_{t}\left(e^{-\lambda t} f^{-2 N} \tilde{E}(u)\right)+\lambda e^{-\lambda t} f^{-2 N} \tilde{E}(u) \\
+\mathcal{O}(N)\left\{e^{-\lambda t} f^{-2 N-1}\left|u^{\prime \prime}\right|^{2}+\partial_{t}\left(e^{-\lambda t} f^{-2 N-2}\left|u^{\prime}\right|^{2}\right)+\lambda e^{-\lambda t} f^{-2 N-2}\left|u^{\prime}\right|^{2}\right\} \\
+\mathcal{O}\left(N^{2}\right) e^{-\lambda t} f^{-2 N-3}\left|u^{\prime}\right|^{2} \\
+\mathcal{O}(N)\left\{\partial_{t}\left(e^{-\lambda t} f^{-2 N-4}|u|^{2}\right)+\lambda e^{-\lambda t} f^{-2 N-4}|u|^{2}\right\} \\
+\mathcal{O}\left(N^{2}\right) e^{-\lambda t} f^{-2 N-5}|u|^{2}+\mathcal{O}(N) e^{-\lambda t} f^{-2 N-1} t a(\xi)\left|u^{\prime}\right|^{2} \\
+\mathcal{O}(N)\left\{\partial_{t}\left(e^{-\lambda t} f^{-2 N-2} t a(\xi)|u|^{2}\right)+\lambda e^{-\lambda t} f^{-2 N-2} t a(\xi)|u|^{2}\right\} \\
+\mathcal{O}\left(N^{2}\right) e^{-\lambda t} f^{-2 N-3} t a(\xi)|u|^{2} \\
-\mathcal{O}(N) e^{-\lambda t} f^{-2 N-2} a(\xi)|u|^{2}-e^{-\lambda t} f^{-2 N} a(\xi)\left|u^{\prime}\right|^{2} \\
+2 e^{-\lambda t} f^{-2 N}\left(t^{2} a_{3}(t, \xi) \operatorname{Im}\left(u \bar{u}^{\prime \prime}\right)+\operatorname{Re} b_{1}(t, \xi) u \bar{u}^{\prime \prime}\right) .
\end{gathered}
$$

There are four "error" terms, all written in the last two lines of (3.6). We deal first with the term containing $u^{\prime}$, the second term term in the second line from below. Neglecting the exponential term, we would like to estimate $f^{-2 N} a(\xi)$ by $f^{-2 N-3}+f^{-2 N-1} t a(\xi)$. First we would like to prove an inequality of the form

$$
\frac{f^{-2 N-3}}{1+a(\xi)}+t f^{-2 N-1} \geq \alpha f^{-2 N}
$$


with a positive constant $\alpha$. Dividing by $f^{-2 N-3}$, the proof is reduced to the inequality

$$
\frac{1}{1+a(\xi)}+t f^{2} \geq \alpha f^{3}
$$

Now

$$
f^{3}=t^{3}+\frac{1}{1+a(\xi)}+\frac{3 t^{2}}{(1+a(\xi))^{1 / 3}}+\frac{3 t}{(1+a(\xi))^{2 / 3}},
$$

while on the left hand side we have

$$
\frac{1}{1+a(\xi))}+t^{3}+\frac{2 t^{2}}{(1+a(\xi))^{1 / 3}}+\frac{t}{(1+a(\xi))^{2 / 3}}
$$

The terms on both sides are the same, so that if we choose $\alpha$ suitably, (3.7) ensues. Thus we deduce

$$
\alpha f^{-2 N} a(\xi) \leq t a(\xi) f^{-2 N-1}+\frac{a(\xi)}{(1+a(\xi)} f^{-2 N-3} \leq t a(\xi) f^{-2 N-1}+f^{-2 N-3} .
$$

Next let us treat the first term in the second line from below in (3.6). We want to estimate $f^{-2 N-2} a(\xi)$ with $f^{-2 N-5}+f^{-2 N-3} t a(\xi)$. This is very easy, since the coefficients of the terms containing $|u|^{2}$ in (3.6) grow as $N^{2}$, and a small portion of them may absorb $\mathcal{O}(N)$. Now the inequality

$$
f^{-2 N-5}+f^{-2 N-3} t a(\xi) \geq \alpha f^{-2 N-2} a(\xi)
$$

is obtained from (3.8), dividing by $f^{2}$.

Now we pass to the analysis of the last term in the last line of (3.6). First we deduce

$$
\operatorname{Re}\left(b_{1}(t, \xi) u \bar{u}^{\prime \prime}\right)=\operatorname{Re} b_{1}(t, \xi) \operatorname{Re}\left(u \bar{u}^{\prime \prime}\right)-\operatorname{Im} b_{1}(t, \xi) \operatorname{Im}\left(u \bar{u}^{\prime \prime}\right) .
$$

To deal with the term involving $\operatorname{Re} b_{1}(t, \xi)$, we use the equality

$$
2 \operatorname{Re}\left(u \bar{u}^{\prime \prime}\right)=\partial_{t} 2 \operatorname{Re}\left(u \bar{u}^{\prime}\right)-2\left|u^{\prime}\right|^{2}
$$

The term with $\left|u^{\prime}\right|^{2}$ be be treated as above since $\left|b_{1}(t, \xi)\right| \leq C \delta_{0} a(\xi)$. To study the term with $\operatorname{Re}\left(u \bar{u}^{\prime}\right)$, we write

$$
\begin{array}{r}
e^{-\lambda t} f^{-2 N} \operatorname{Re}\left(b_{1}(t, \xi) \operatorname{Re}\left(u \bar{u}^{\prime}\right)\right) \\
=\partial_{t}\left(e^{-\lambda t} f^{-2 N} \operatorname{Re} b_{1}(t, \xi) \operatorname{Re}\left(u \bar{u}^{\prime}\right)\right)+\lambda e^{-\lambda t} f^{-2 N} \operatorname{Re} b_{1}(t, \xi) \operatorname{Re}\left(u \bar{u}^{\prime}\right) \\
+2 N f^{-2 N-1} \operatorname{Re} b_{1}(t, \xi) \operatorname{Re}\left(u \bar{u}^{\prime}\right)+e^{-\lambda t} f^{-2 N} \operatorname{Re} b_{1, t}(t, \xi) \operatorname{Re}\left(u \bar{u}^{\prime}\right)=\partial_{t}(\ldots)+I+I I+I I I .
\end{array}
$$

There are three terms on the right hand side of (3.9). Consider I. Applying the Cauchy-Schwartz inequality and $\left|b_{1}(t, \xi)\right| \leq C|\xi|^{2}$, we obtain

$$
\begin{array}{r}
\lambda\left|e^{-\lambda t} f^{-2 N} \operatorname{Re} b_{1}(t, \xi) \operatorname{Re}\left(u \bar{u}^{\prime}\right)\right| \\
\leq C \lambda \delta_{0}^{-1}\left[\varepsilon e^{-\lambda t} f^{-2 N+1} a(\xi)\left|u^{\prime}\right|^{2}+\frac{1}{\varepsilon} e^{-\lambda t} f^{-2 N-1} a(\xi)|u|^{2}\right] \\
\leq C \lambda \delta_{0}^{-1} \varepsilon \alpha^{-1} e^{-\lambda t}\left[f^{-2 N} t a(\xi)\left|u^{\prime}\right|^{2}+f^{-2 N-2}\left|u^{\prime}\right|^{2}\right] \\
+\frac{C \lambda}{\varepsilon} \delta_{0}^{-1} \alpha^{-1} e^{-\lambda t}\left[f^{-2 N-2} t a(\xi)|u|^{2}+f^{-2 N-4}|u|^{2}\right],
\end{array}
$$


where $\varepsilon>0$ is a small positive constant, to be chosen below. Taking $C \delta_{0}^{-1} \alpha^{-1} \varepsilon<1 / 2$, we may estimate the term with $f^{-2 N} t a(\xi)\left|u^{\prime}\right|^{2}$ by $f^{-2 N} \tilde{E}(u)$. Next $\frac{C \lambda}{\varepsilon} \delta_{0}^{-1} \alpha^{-1} e^{-\lambda t} f^{-2 N-2} t a(\xi)|u|^{2}$ can be absorbed by the corresponding term in (6) with large $N$ and the same is true for the term with $f^{-2 N-4}|u|^{2}$. The analysis of $I I I$ is similar and simpler.

To handle $I I$, we use the inequality

$$
I I \leq C_{1}^{2} \delta^{-1} e^{-\lambda t} f^{-2 N} a(\xi)\left|u^{\prime}\right|^{2}+4 N^{2} \delta f^{-2 N-2} a(\xi)|u|^{2},
$$

where $C_{1}=C \delta_{0}^{-1}$ and $\beta>0$ is a small constant.

The latter term in the above line is similar to the first in the last line of (3.6); the only difference is the factor in front, which is bigger here. However, remarking that all the terms containing $|u|^{2}$ in (3.6) have also $\mathcal{O}\left(N^{2}\right)$, it is clear that choosing $\delta$ suitably small, but finite and independent of $u, N$ and $\lambda$, will allow us to conclude by arguing as above. The fist summand on the other hand is similar to the middle term in the last line of (3.6): $C_{1}$ is real and depends on the lower order terms, $\delta$ is fixed. This is estimated as we did before, provided that $N$ is large enough.

Next we turn to the term containing $-\operatorname{Im} b_{1}(t, \xi) \operatorname{Im}\left(u \bar{u}^{\prime \prime}\right)$ containing $\operatorname{Im} b_{1}(t, \xi)$. We remark that $\operatorname{Im}\left(u \bar{u}^{\prime \prime}\right)=\partial_{t}\left(u \bar{u}^{\prime}-u^{\prime} \bar{u}\right)$, so that we obtain two terms which can be discussed almost verbatim as before. This might require enlarging $N$.

Finally, consider the term

$$
2 e^{-\lambda t} f^{-2 N} t^{2} a_{3}(t, \xi) \operatorname{Im}\left(u \bar{u}^{\prime \prime}\right) \geq-C_{1} e^{-\lambda t} f^{-2 N} t^{4}\left(1+|\xi|^{2}\right)^{3}|u|^{2}-e^{-\lambda t} f^{-2 N}\left|u^{\prime \prime}\right|^{2} .
$$

The last term in the right hand side can be treated as above, however the first one cannot be absorbed by other positive terms taking $N$ large enough. Consequently, in (3.6) we obtain an upper bound on the left by

$$
e^{-\lambda t} f^{-2 N+1}|\hat{P} u|^{2}+C_{1} t^{4} e^{-\lambda t} f^{-2 N}\left(1+|\xi|^{2}\right)^{3}|u|^{2}
$$

Now assume $0 \leq s<T \leq 1$ and $v=D_{t} v=D_{t}^{2} v=0$ when $t=s$. We integrate from $t$ to $T$ w.r.t. to the time variable $s$. Thus yields some integrals $\int_{t}^{T}(\ldots) d$.$s and terms$

$$
\begin{gathered}
e^{-\lambda T} f^{-2 N}(T, \xi) \tilde{E}(u(T, \xi)) \\
+\mathcal{O}(N)\left[e^{-\lambda T} f^{-2 N-2}(T, \xi)\left|u^{\prime}(T, \xi)\right|^{2}+e^{-\lambda T} f^{-2 N-4}(T, \xi)|u(T, \xi)|^{2}\right] \\
+\mathcal{O}(N) e^{-\lambda T} f^{-2 N-2}(T, \xi) T a(\xi)|u(T, \xi)|^{2} \\
+2 e^{-\lambda T} f^{-2 N}(T, \xi) \operatorname{Re} b(T, \xi) \operatorname{Re}\left(u \bar{u}^{\prime}\right)(T, \xi) \\
-2 e^{-\lambda T} f^{-2 N}(T, \xi) \operatorname{Im} b(T, \xi)\left(u \bar{u}^{\prime}-\bar{u}^{\prime} u\right)(T, \xi) .
\end{gathered}
$$

Concerning the boundary terms, only the last three terms in the above sum has no positive sign. We have no coefficient $\lambda$ before them and these terms can be treated by the above argument using the positive terms and choosing $N$ large enough. Next we integrate with respect to $\xi$ in $\mathbb{R}^{n}$ and we replace the $L^{2}\left(\mathbb{R}_{\xi}^{n}\right)$ norms by $L^{2}\left(\mathbb{R}_{x}^{n}\right)$ norms. Moreover, we have the obvious inequalities

$$
\begin{gathered}
f^{-1}=\frac{(1+a(\xi))^{1 / 3}}{t(1+a(\xi))^{1 / 3}+1} \leq(1+a(\xi))^{1 / 3} \leq C_{2}\left(1+|\xi|^{2}\right)^{1 / 3}, t \geq 0, \\
f^{-1} \geq 1 / 2,0 \leq t<T \leq 1 .
\end{gathered}
$$


Then

$$
\begin{gathered}
C_{1} t^{4} \int_{t}^{T} \int e^{-\lambda s} f^{-2 N}\left(1+|\xi|^{2}\right)^{3}|u|^{2} d \xi d s \leq C_{2} t^{4} \int_{t}^{T} \int e^{-\lambda s}\left(1+|\xi|^{2}\right)^{2 N / 3+3}|u|^{2} d \xi d s \\
\leq C_{3} t^{4} \int_{t}^{T} e^{-\lambda s}\|v\|_{(2 N / 3+3)}^{2} d s
\end{gathered}
$$

where $\|\cdot\|_{(s)}$ is the $H_{(s)}$ norm in $\mathbb{R}^{n}$ for fixed $s$. Now for $v$ and $0<t \leq T$ and small $T$ we may apply the energy estimates for strictly hyperbolic operators (see Section 23.2 and the proof of Lemma 23.2.1 in [5]). Taking into account Lemma 2, we get

$$
\int_{t}^{T} e^{-\lambda s}\|v\|_{(2 N / 3+3)}^{2} d s \leq \frac{C_{N}}{t^{2}} \int_{t}^{T} e^{-\lambda s}\|P v\|_{(2 N / 3+1)}^{2} d s .
$$

We introduce $U_{1}(s, \xi)=\left(1+|\xi|^{2}\right)^{1 / 2} u(s, \xi)$ and observe that $U_{1}$ satisfies the same initial conditions on $s=t$ as $u$ and

$$
\hat{P} U_{1}=\left(1+|\xi|^{2}\right)^{1 / 2} \hat{P} u
$$

Finally, we obtain the following

Theorem 2. Let $v \in C_{0}^{\infty}\left(\mathbb{R}_{t} \times \mathbb{R}^{n}\right)$ and let $v(s, x)=D_{t} v(s, x)=D_{t}^{2} v(s, x)=0$ for $s=t$. Let $0 \leq t<T \leq 1$ Then for $T$ small enough and for an integer $N$ and $\lambda>\lambda_{0}$ depending on the lower order terms $b\left(t, D_{x}\right)$ we have the estimate

$$
\lambda \int_{t}^{T} e^{-\lambda s}\left(\left\|D_{t}^{2} v\right\|_{(1)}^{2}+\left\|D_{t} v\right\|_{(2)}^{2}+\|v\|_{(2)}^{2}\right) d s \leq C(N) \int_{t}^{T} e^{-\lambda s}\|P v\|_{(2 N / 3+2)}^{2} d s .
$$

Now will treat the estimates for functions $v \in C_{0}^{\infty}\left(\mathbb{R}_{t} \times \mathbb{R}^{n}\right)$ with initial data

$$
v(T, x)=D_{t} v(T, x)=D_{t}^{2} v(T, x)=0 .
$$

To do this we multiply $\hat{P} u$ by $-e^{\lambda t} f^{2 N} \bar{u}^{\prime \prime}$ and repeating the above argument, we obtain for $0 \leq t<$ $T \leq 1$

$$
\begin{gathered}
e^{\lambda t} f^{2 N+1}|\hat{P} u|^{2} \geq-\partial_{t}\left(e^{\lambda t} f^{2 N} \tilde{E}(u)\right)+\lambda e^{\lambda t} f^{2 N} \tilde{E}(u) \\
+\mathcal{O}(N)\left\{e^{\lambda t} f^{2 N-1}\left|u^{\prime \prime}\right|^{2}-\partial_{t}\left(e^{\lambda t} f^{2 N-2}\left|u^{\prime}\right|^{2}\right)+\lambda e^{\lambda t} f^{2 N-2}\left|u^{\prime}\right|^{2}\right\} \\
+\mathcal{O}\left(N^{2}\right) e^{\lambda t} f^{2 N-3}\left|u^{\prime}\right|^{2} \\
+\mathcal{O}(N)\left\{-\partial_{t}\left(e^{\lambda t} f^{2 N-4}|u|^{2}\right)+\lambda e^{\lambda t} f^{2 N-4}|u|^{2}\right\} \\
+\mathcal{O}\left(N^{2}\right) e^{\lambda t} f^{2 N-5}|u|^{2} \\
-2 N e^{\lambda t} f^{2 N-1} t a(\xi)\left|u^{\prime}\right|^{2}+e^{\lambda t} f^{2 N} a(\xi)\left|u^{\prime}\right|^{2} \\
+2 e^{\lambda t} f^{2 N} t^{2} a_{3}(t, \xi) \operatorname{Im}\left(u \bar{u}^{\prime \prime}\right)-2 e^{\lambda t} f^{2 N} \operatorname{Re} b_{1}(t, \xi) u \bar{u}^{\prime \prime} .
\end{gathered}
$$

Now we assume $0 \leq s<T \leq 1$ and let $v=D_{t} v=D_{t}^{2} v=0$ when $s=T$. We integrate from $t$ to $T$ with respect to the time variable $s$ and we treat the boundary terms with $s=t$ as above, while the "error" terms are handled in the same way as in the case with initial data on $s=t$. Thus we obtain a priori estimate involving the "weights" $f^{2 N-k}\left(D_{x}\right),-1 \leq k \leq 5$. On the other hand,

$$
f^{2 N+1} \leq(t+1)^{2 N+1}, 0 \leq t<T \leq 1,
$$




$$
f^{2 N} \geq \frac{1}{(1+a(\xi))^{2 N / 3}} \geq B_{N}\left(1+|\xi|^{2}\right)^{-2 N / 3} .
$$

We introduce $U_{N}(s, \xi)=\left(1+|\xi|^{2}\right)^{(2 N+2) / 3} u(t, \xi)$ and observe that $U_{N}$ satisfies the same initial conditions on $s=T$ as $u$ and

$$
\hat{P} U_{N}=\left(1+|\xi|^{2}\right)^{(2 N+2) / 3} \hat{P} u .
$$

Thus we deduce the following

Theorem 3. Let $v \in C_{0}^{\infty}\left(\mathbb{R}_{t} \times \mathbb{R}^{n}\right)$ and let $v(s, x)=D_{t} v(s, x)=D_{t}^{2} v(s, x)=0$ for $s=T$. Let $0 \leq t<T \leq 1$. Then for $T$ small enough and for an integer $N$ and $\lambda>\lambda_{0}$ depending on the lower order terms $b\left(t, D_{x}\right)$ we have the estimate

$$
\lambda \int_{t}^{T} e^{\lambda s}\left(\left\|D_{t}^{2} v\right\|_{(2 / 3)}^{2}+\left\|D_{t} v\right\|_{(4 / 3)}^{2}+\|v\|_{(2)}^{2}\right) d s \leq C_{1}(N, T) \int_{t}^{T} e^{\lambda s}\|P v\|_{(2 N / 3+2)}^{2} d s,
$$

where $\|\cdot\|_{(m)}$ is the $H_{(m)}$ norm in $\mathbb{R}^{n}$ for fixed $s$.

From Theorems 2 and 3 we conclude in a standard way that the Cauchy problem for $P$ is well posed.

\section{Operators With COEFFCIENTs DEPENDing ON $t$ AND $x$}

We sketch briefly some ideas for the analysis of the case when we have operators with coefficients depending on $t$ and $x$.

First consider a scaling $t=\varepsilon^{2 / 3} s, x=\varepsilon y, \varepsilon>0$. Multiplying by $\varepsilon^{2}$, we obtain an operator

$$
\begin{gathered}
P=D_{s}^{3}-s a_{2}\left(\varepsilon^{2 / 3} s, \varepsilon y, D_{y}\right) D_{s}+B_{2}\left(\varepsilon^{2 / 3} s, \varepsilon y, D_{y}\right) \\
+\varepsilon^{1 / 3}\left[s a_{1}\left(\varepsilon^{2 / 3} t, \varepsilon y, D_{y}\right) D_{s}^{2}+s^{2} a_{3}\left(\varepsilon^{2 / 3} s, \varepsilon y, D_{y}\right)+B_{1}\left(\varepsilon^{2 / 3} s, \varepsilon y, D_{y}\right) D_{s}\right]+\varepsilon C_{1}(\ldots) .
\end{gathered}
$$

Our final purpose is to choose $\varepsilon=\mathcal{O}\left(\frac{1}{N}\right)$, where $N$ is a big fixed integer related to lower order terms as in the case treated in Section 3. With this choice of $\varepsilon$ we are going to study the Cauchy problem for sufficiently small $t>0$. This is enough since for $t>0$ our operator is strictly hyperbolic.

We cannot apply Fourier transform and moreover it is convenient to employ a suitable class of pseudodifferential operators. Notice that $f=t+\left(1+a_{2}(t, x, \xi)\right)^{-1 / 3}$ is a symbol in the class $S_{1,2 / 3}^{0}$, when derivatives with respect to $t$ are considered, but is in the class $S_{1,0}^{0}$ if $t$ is just a parameter and no derivatives with respect to $t$ are involved.

Let $\langle\xi\rangle^{2}=1+|\xi|^{2}$ and let

$$
g_{(x, \xi)}=|d x|^{2}+\langle\xi\rangle^{-2}|d \xi|^{2}
$$

be the classical slowly varying $(1,0)$ - metric. We need also the dilated metric

$$
g_{(x, \xi)}^{\varepsilon}=\varepsilon^{2}|d x|^{2}+\langle\xi\rangle^{-2}|d \xi|^{2} .
$$

Define the following "order" function

$$
m_{N}^{t, \mu}(x, \xi)=f^{-N}(t, \xi)\langle\xi\rangle^{\mu / 2},
$$

where $N$ is a large integer and $\mu$ is any real number. Then we may define the class $S\left(m_{N}^{t, \mu}, g\right)$ of symbols in the standard way. We point out explicitly that $t$ is just a parameter and at this level we may omit it in our notation. We have

Proposition 1. $f^{-N}(t, \xi) \in S\left(m_{N}^{t, 0}, g\right)$. 
We have also

Proposition 2. Let $c(x, \xi) \in S^{\mu}(1, g)$ be a classical symbol of order $\mu$. Then $f^{-N}(t, \xi) \#_{x} c(x, \xi)=$ $b_{t}(x, \xi)$, where $b_{t} \in S\left(m_{N}^{t, \mu}, g\right)$. Here $\#_{x}$ denotes the operation of formal asymptotic composition $g \#_{x} c=\sum_{|\alpha| \geq 0} \frac{1}{\alpha !} \partial_{\xi}^{\alpha} g(x, \xi) D_{x}^{\alpha} c(x, \xi)$.

To examine the lower order terms we need to handle the term

$$
f^{-N}\left(t, D_{x}\right) b\left(\varepsilon^{2 / 3} t, \varepsilon x, D_{x}\right) f^{N}\left(t, D_{x}\right),
$$

$b\left(t, x, D_{x}\right)$ being a second order pseudodifferential operator. We deduce that

$$
B_{N}=f^{-N}(t, \xi) \#_{x} b\left(\varepsilon^{2 / 3} t, \varepsilon x, \xi\right)\left(1+|\xi|^{2}\right)^{-1} f^{N}(t, \xi) \in S\left(m_{N}^{t, 0}, g\right)
$$

but we need to estimate the $L^{2}$ norm of the operator $B_{N}$ and for this reason we take $\varepsilon$ to be of order $\mathcal{O}\left(\frac{1}{N}\right)$. Therefore in the calculus of lower order terms of $B_{N}$ the powers of $N$ are compensated by the powers of $\varepsilon$. Moreover, we may write the composition of symbols $B_{N}$ by using a finite sum and an integral representation of the remainder introduced by J.M. Bony [2].

The details of the analysis of the operators with variable coefficients depending on $(t, x)$ will be given in a paper in preparation [1].

\section{REFERENCES}

[1] E. Bernardi, A. Bove, V. Petkov, The Cauchy problem for effectively hyperbolic operators with triple characteristics, in preparation.

[2] J. M. Bony, Sur l'inégalité de Fefferman-Phong, Séminaire EDP, Ecole Polytechnique, 1998-1999.

[3] J. Chazarain, Opérateurs hyperboliques à caractéristiques de multiplicité constante, Ann. Institut Fourier (Grenoble), 24 (1974), 173-202.

[4] H. Flashka and G. Strang, The correctness of the Cauchy problem, Adv. in Math. 6 (1971), 347-379.

[5] L. Hörmander, Cauchy problem for differential operators with double characteristics, J. Analyse Math. 32 (1977), 118-196.

[6] L. Hörmander, Analysis of Linear Partial Differential Operators, III, Springer-Verlag, 1985, Berlin.

[7] V. Ja. Ivrii and V. M. Petkov, Necessary conditions for the Cauchy problem for non-strictly hyperboilic equations to be well posed, Uspehi Mat. Nauk, 29: 5 (1974), 1-70 (in Russian), English translation: Russian Math. Surveys, 29:5 (1974), 3-70.

[8] V. Ivrii, Sufficient conditions for regular and completely regular hyperbolicity, Trudy Moskov Mat. Obsc.,33 (1976), 3-66 (in Russian), English translation: Trans. Moscow Math. Soc. 1 (1978), 165.

[9] N. Iwasaki, The Cauchy problem for effectively hyperbolic equations (a standard type), Publ. RIMS Kyoto Univ. 20 (1984), 551-592.

[10] N. Iwasaki, The Cauchy problem for effectively hyperbolic equations (general case), J. Math. Kyoto Univ. 25 (1985), 727-743.

[11] R. Melrose, The Cauchy problem for effectively hyperbolic operators, Hokkaido Math. J. 12 (1983), 371-391.

[12] T. Nishitani, Local energy integrals for effectively hyperbolic operators, I, II, J. Math. Kyoto Univ. 24 (1984), 623-658 and 659-666.

[13] T. Nishitani, The effectively Cauchy problem in The Hyperbolic Cauchy Problem, Lecture Notes in Mathematics, 1505, Springer-Verlag, 1991, pp. 71-167.

[14] O. A. Oleinik, On the Cauchy problem for weakly hyperbolic equations, Comm. Pure Appl. Math. 23 (1970), 569-586.

[15] M. R. Spiegel, J. Liu, Mathematical handbook of formulas and tables, McGraw-Hill, Second Edition, 1999.

Dipartimento di Matematica per le Scienze Economiche e Sociali, Università di Bologna, Viale Filopanti 5, 40126 Bologna, Italia

E-mail address: bernardi@economia.unibo.it 
Dipartamento di Matematica, Università di Bologna, Piazza di Porta S. Donato 5, 40126 Bologna, ITALIA

E-mail address: bove@dm.unibo.it

Université Bordeaux I, Institut de Mathématiques de Bordeaux, 351, Cours de la Libération, 33405 TAlence, France

E-mail address: petkov@math.u-bordeaux1.fr 